\title{
Long-Term Lead Performance for Vagus Nerve Stimulation: Low Rate of Complications and Failures
}

\author{
Inder S. Anand ${ }^{1 *}$, Imad Libbus², and Lorenzo A. DiCarlo² \\ ${ }^{1}$ Division of Cardiology, University of Minnesota, Minneapolis, Minnesota, USA \\ ${ }^{2}$ LivaNova USA, Inc., Houston, Texas, USA
}

\begin{abstract}
Background: Vagus nerve stimulation (VNS) has been shown to improve cardiac function and heart failure symptoms. The VITARIA System provides chronic stimulation through a self-sizing, atraumatic lead placed around the cervical vagus nerve. The lead is identical to the predecessor M304 lead, which has been implanted in patients since 2009 for treatment of epilepsy and depression. Its long-term performance has not been previously reported. Methods: All leads implanted in the United States for any indication were included in this analysis. All available data on lead explants, replacements, and customer complaints were used to identify failures. Lead survival was defined as likelihood of the implanted lead remaining implanted and performing as intended. Results: The M304 lead has been part of 31,000 implantations, with 72,100 device-years of patient exposure. In 11,000 patients, 99.4\% of leads remained implanted and performing as intended after 1 year. At 7 years, $95.7 \%$ of leads performed as intended. Lead failure is rare, with common causes being infection $(0.87 \%)$ and vocal cord dysfunction $(0.68 \%)$. Conclusions: The M304 VNS lead has been used for neuromodulation in over 30,000 patients for over 70,000 device-years. Cumulative lead survival has exceeded design requirements and has low rates of complications and failures.
\end{abstract}

Keywords: autonomic regulation therapy; vagus nerve stimulation; heart failure; device performance

Citation: Anand, I. S., Libbus, I., \& DiCarlo, L. A. (2020). Long-term lead performance for vagus nerve stimulation: Low rate of complications and failures. NeuroRegulation, 7(1), 26-29. https://doi.org/10.15540/nr.7.1.26

*Address correspondence to: Inder S. Anand, MD, VA Medical Center, Cardiology 111-C, One Veterans Drive, Minneapolis, MN 55417, USA. Email: anand001@umn.edu

Copyright: (c) 2020. Anand et al. This is an Open Access article distributed under the terms of the Creative Commons Attribution License (CC-BY).

\section{Introduction}

Heart failure (HF) is characterized by hemodynamic abnormalities that result in an imbalance between an increase in sympathetic activity and withdrawal of parasympathetic tone. This contributes to the progression of $\mathrm{HF}$ and an increase in the risk of mortality and morbidity independent of ejection fraction (EF) and ventricular arrhythmias.

Autonomic regulation therapy (ART) is a novel investigational approach for the management of HF that uses cervical vagus nerve stimulation (VNS) to increase parasympathetic activity and help restore autonomic balance. ART is delivered using chronic stimulation through a self-sizing lead that is placed on the cervical vagus nerve (CVN) without requiring any
Edited by:

Rex L. Cannon, PhD, SPESA Research Institute, Knoxville, Tennessee, USA

Reviewed by:

Rex L. Cannon, PhD, SPESA Research Institute, Knoxville, Tennessee, USA

Randall Lyle, PhD, Mount Mercy University, Cedar Rapids, lowa, USA 
2009 for the treatment of drug-refractory epilepsy (DRE) and treatment-resistant depression (TRD; Groves \& Brown, 2005; Morris \& Mueller, 1999). In

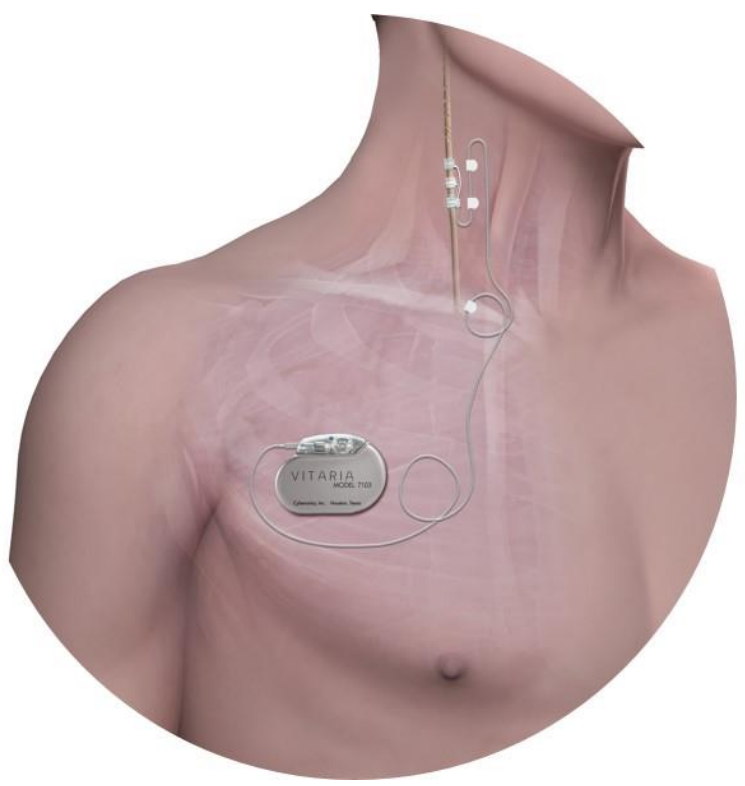

this study, we provide findings of a survival analysis, performed to evaluate the long-term performance of the Model 304 PerenniaFLEX lead.

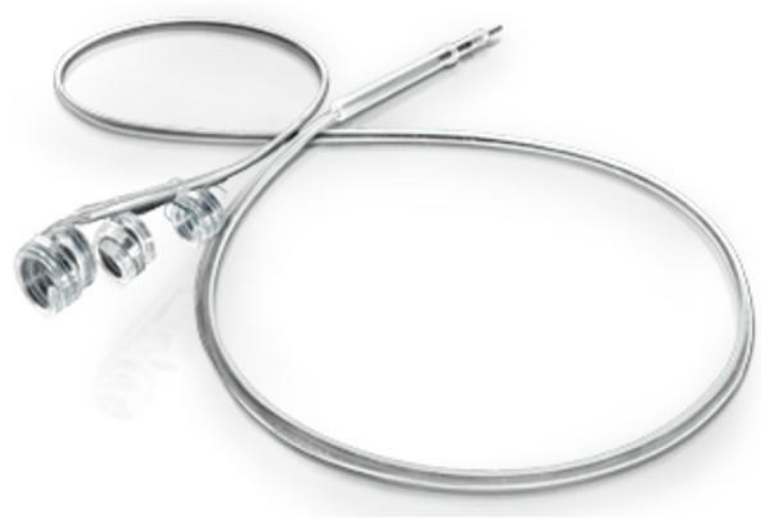

Figure 1. The VITARIA autonomic regulation therapy (ART) system implanted on the right cervical vagus nerve (left), which includes the VITARIA Model 7304 lead (right).

\section{Methods}

\section{Data Collection}

The LivaNova quality system was used to retrieve and to evaluate lead data. The system includes a device tracking system, which tracks device shipments and implant registration forms that are completed by the implanting physician or hospital staff. LivaNova collects data on device explants, device replacements, returned product analyses, and customer complaints through multiple sources, including voluntary product return, complaint reporting, and device tracking. These data are collected and used to identify failures and out-ofspecification conditions. The implant registration forms allow implantable products to be tracked at the patient level from the time of device implant to the time of device explant or patient death. All leads that were registered as implanted in the United States for any indication were included in the analysis, and all data available through December 31, 2018, was analyzed. The analysis complied with the Declaration of Helsinki (World Medical Association, 1996).

To minimize the potential of underreporting of patient deaths, active surveillance was conducted of data obtained from the United States Social Security Administration Death Master File and the Center for Disease Control National Death Index. Those patients without a known Social Security Number were not included in the survival calculations.

\section{Survival Probability Calculations}

Lead survival probability was defined as the likelihood of the implanted device remaining implanted and performing as intended at a specific point in the product's service life. The actuarial method was used to estimate survival probability at any time interval (Tolley, Barnes, \& Freeman, 2016). Device survival plots use the number of successfully functioning units divided by the total number of units. The cumulative survival probability at a point in time is the product of the survival probabilities for all preceding time intervals. The exponential Greenwood's formula was used to estimate the standard error of the calculated survival probabilities and $95 \%$ confidence intervals (Cl; Kalbfleisch \& Prentice, 1980).

\section{Results}

At the time of this analysis, there have been 31,000 registered implants of the PerenniaFLEX Model 304 
VNS lead, with a cumulative implant follow-up duration of 72,100 device-years. It is estimated that 26,400 of these devices are still active. Survival status for 11,000 patients was obtainable from public records, and these implants were included in the survival calculations.

Cumulative survival of the implanted leads over time is shown in Table 1 and Figure 2. After 1 year, 99.4\% of leads remained implanted and performed as intended $(95 \% \mathrm{Cl}[99.2,99.5])$. After 5 years and 7 years, $97.1 \%(95 \% \mathrm{Cl}[96.7,97.5 \%])$ and $95.7 \%(95 \%$ Cl $[95.1,96.2])$ of implanted leads remained implanted and performed as intended. This survival performance exceeds the design requirement for the Model 304 lead (Figure 2).

\section{Table 1}

Cumulative survival of the Model 304 lead over various follow-up intervals, shown as a percentage with $95 \%$ confidence intervals.

\begin{tabular}{ccccccc}
\hline & At implant & 1 year & 2 year & 3 year & 5 year & 7 year \\
\hline $\begin{array}{c}\text { Cumulative } \\
\text { Survival (\%) }\end{array}$ & $100 \%$ & $99.4 \%$ & $98.8 \%$ & $98.4 \%$ & $97.1 \%$ & $95.7 \%$ \\
$\begin{array}{c}\text { Number of } \\
\text { Patients }\end{array}$ & 11,000 & 9,700 & 8,500 & 7,200 & 4,600 & 1,800 \\
\hline
\end{tabular}

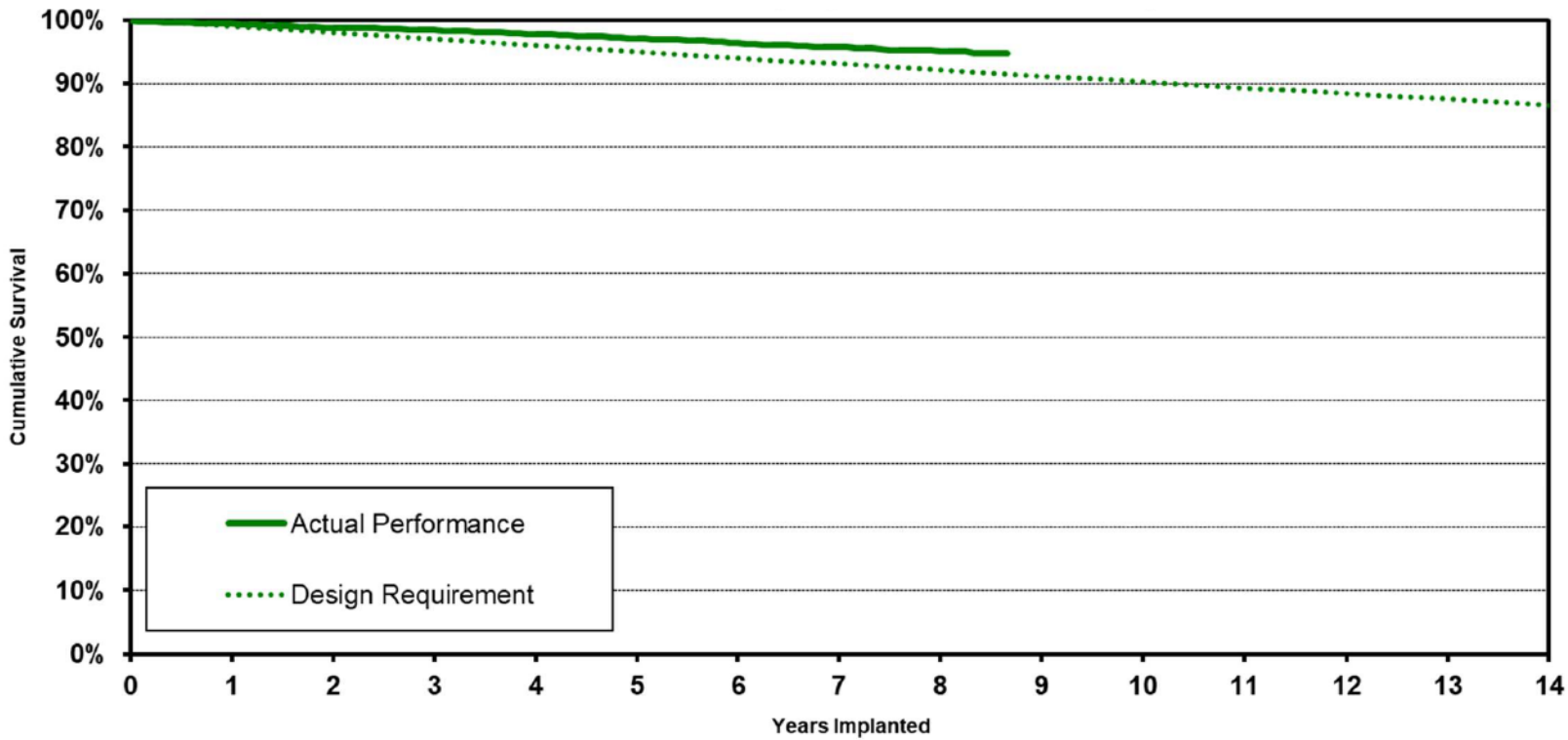

Figure 2. Cumulative survival of the Model 304 lead for US patients with known SSN (solid line) compared to design requirements (dotted line).

Lead complications and failures have been rare, with the most common complications being infection $(0.87 \%)$, vocal cord dysfunction $(0.68 \%)$, lead protrusion $(0.36 \%)$, and lead extrusion $(0.27 \%)$.

\section{Discussion}

There are several similarities in how VNS is administered for the treatment of DRE, TRD, and HF.
VNS systems used in all these conditions include an implantable pulse generator, an electrode lead that surrounds the CVN. An external programming system is used to change the generator settings for stimulating the CVN. The electrode is placed around the vagus nerve without requiring intraoperative mapping. The pulse generator and lead deliver electrical stimulation to axons in the CVN. The axons are approximately $80 \%$ afferent and $20 \%$ 
parasympathetic preganglionic efferents (Jänig, 2006).

VNS is administered to the left CVN using the Model 304 lead with bidirectional open-loop delivery for the management of DRE and TRD. For HF, VNS has been administered to the left or right CVN using the Model 7304 lead with bidirectional open-loop delivery that is directed preferentially toward peripheral vagal efferents that control cardiovascular function. In investigational studies in HFrEF, VNS has utilized a relatively lower amplitude (1.5 to $3 \mathrm{~mA}$ current) and pulse frequency $(5-10 \mathrm{~Hz})$ than is used for DRE. Model 304 lead for DRE and TRD and Model 7304 lead for $\mathrm{HF}$ are identical in materials and manufacture. The functional difference between these leads is in the polarity of the electrodes. In DRE and TRD, the cathode is positioned cranially, and in $\mathrm{HF}$, the cathode is positioned caudally (Anand, Konstam, Ardell, Libbus, \& DiCarlo, 2019; BenMenachem, 2002).

Implanted lead performance is continually tracked during clinical studies and as part of the LivaNova postmarket surveillance process to identify device failure and to determine causes and potential out-ofspecification conditions. Based upon the current analysis, chronic VNS using Model 304 appears to be associated with a satisfactory long-term safety and performance profile.

\section{Conclusions}

Evaluation of the long-term performance of the Model 304 VNS lead, used in the treatment of DRE and TRD, has demonstrated excellent cumulative survival that exceeded its design requirements, and its use for neuromodulation has been associated with low rates of complications and lead failures. The VITARIA System for the treatment of HF includes the Model 7304 VNS lead, which is identical in its materials and manufacture and is being utilized in the ANTHEMHFrEF pivotal study.

\section{Author Note}

No direct funding was provided for this study.

\section{Author Disclosure}

Dr. Anand is contracted to LivaNova as a cardiovascular consultant. Dr. Libbus and Dr. DiCarlo are employees and shareholders of LivaNova.

\section{References}

Anand, I. S., Konstam, M. A., Ardell, J. L., Libbus, I., \& DiCarlo, L. (2019). Neuromodulation for drug-refractory epilepsy and chronic heart failure: Targets, delivery, composition and titration. International Journal of Neurology and Neurotherapy, 6(2), 091. https://doi.org/10.23937/2378-3001/1410091

Ben-Menachem, E. (2002). Vagus-nerve stimulation for the treatment of epilepsy. The Lancet Neurology, 1(8), 477-482. https://doi.org/10.1016/S1474-4422(02)00220-X

Groves, D. A., \& Brown, V. J. (2005). Vagal nerve stimulation: A review of its applications and potential mechanisms that mediate its clinical effects. Neuroscience \& Biobehavioral Reviews, 29(3), 493-500. https://doi.org/10.1016 /j.neubiorev.2005.01.004

Jänig, W. (2006). Functional anatomy of the peripheral sympathetic and parasympathetic system. In The integrative action of the autonomic nervous system: Neurobiology of homeostasis (pp. 13-34). Cambridge, UK: Cambridge University Press.

Kalbfleisch, J. D., \& Prentice, R. L. (1980). The statistical analysis of failure time data. New York, NY: John Wiley \& Sons.

Konstam, M. A., Udelson, J. E., Butler, J., Klein, H. U., Parker, J. D., Teerlink, J. R., ... DiCarlo, L. A. (2019). Impact of autonomic regulation therapy in patients with heart failure: ANTHEM-HFrEF pivotal study design. Circulation: Heart Failure, 12(11), e005879. https://doi.org/10.1161 /circheartfailure.119.005879

Morris, G. L., \& Mueller, W. M. (1999). Long-term treatment with vagus nerve stimulation in patients with refractory epilepsy. Neurology, 53(8), 1731-1735. https://doi.org/10.1212 /WNL.53.8.1731

Premchand, R. K., Sharma, K., Mittal, S., Monteiro, R., Dixit, S., Libbus, I., ... Anand, I. S. (2014). Autonomic regulation therapy via left or right cervical vagus nerve stimulation in patients with chronic heart failure: Results of the ANTHEM-HF trial. Journal of Cardiac Failure, 20(11), 808-816. https://doi.org/10.1016 /j.cardfail.2014.08.009

Premchand, R. K., Sharma, K., Mittal, S., Monteiro, R., Dixit, S., Libbus, I., ... Anand, I. S. (2016). Extended follow-up of patients with heart failure receiving autonomic regulation therapy in the ANTHEM-HF study. Journal of Cardiac Failure, 22(8), 639-642. https://doi.org/10.1016/j.cardfail.2015.11.002

Premchand, R. K., Sharma, K., Mittal, S., Monteiro, R., Libbus, I. DiCarlo, L., ... Anand, I. (2019). Long-term follow-up of reduced ejection fraction heart failure patients receiving autonomic regulation therapy in the ANTHEM-HF pilot study. Journal of the American College of Cardiology, 73(9 Suppl. 1), 770. https://doi.org/10.1016/S0735-1097(19)31378-6

Tolley, H. D., Barnes, J. M., \& Freeman, M. D. (2016). Survival analysis. In M. D. Freeman \& M. P. Zeegers (Eds.), Forensic epidemiology: Principles and practice (pp. 261-284). London, UK: Elsevier Inc.

Received: February 24, 2020

Accepted: March 18, 2020

Published: March 25, 2020 